Mazzi $\mathrm{et} \mathrm{al}^{4}$ showed that local treatment lowered the incidence of scalp abscesses in their hospital.

We found that only two factors were significantly associated with severe scalp changes. Duration of monitoring was the most important factor and the second was parity. First delivery was associated with an increase in severe complications, even after the duration of monitoring was controlled by multivariate analysis. It could be that during the first delivery, which is usually longer, the scalp is more prone to oedema and tissue hypoxia, which contribute to severe scalp changes. It also may well be a chance finding. Okada $e t a l$ found that duration of monitoring and 'high risk indication for monitoring' (especially prematurity) were associated with scalp abscesses. We believe that the severe complications of fetal monitoring are partially preventable. Strict aseptic technique on application of the electrodes is important, as is electrode removal, which should be done with slow spiral movement. Local treatment of small lacerations by shaving the hair and application of antiseptic solution is recommended.

\section{References \\ ${ }^{1}$ Chan WH, Paul RH, Towes J. Intrapartum fetal monitoring: maternal and fetal morbidity and perinatal mortality. Obstet Gynecol 1973;41:7-13. \\ ${ }^{2}$ Plavidal FJ, Werch A. Fetal scalp abscess secondary to intrauterine monitoring. Am J Obstet Gynecol 1976;125:65-70. \\ 3 Okada DM, Chow AW. Neonatal scalp abscess following intrapartum fetal monitoring: prospective comparison of two spiral electrodes. Am J Obstet Gynecol 1977;127:875-878. \\ 4 Mazzi E, Herrera A, Bergman MA. Prevention of scalp abscesses secondary to fetal scalp monitoring. J Pediatr 1977;90: 664-5. \\ 5 Okada DM, Chow AW, Bruce VT. Neonatal scalp abscess and fetal monitoring: factors associated with infection. Am J Obstet Gynecol 1977;129:185-9.}

Correspondence to Dr S Ashkenazi, Department of Neonatology, Beilinson Medical Center, Petah Tiqva 49 100, Israel.

Received 24 September 1984

\title{
Carcinoid syndrome: an unusual cause of diarrhoea
}

\author{
M D KING, D G YOUNG, I M HANN, AND W J A PATRICK \\ Departments of Child Health, Paediatric Surgery, Haematology, and Pathology, Royal Hospital for Sick \\ Children, Glasgow
}

SUMMARY A 9 year old girl underwent laparotomy because of intermittent diarrhoea, present since infancy. Histology of a mass at the head of pancreas and multiple hepatic nodules suggested an apudoma. Plasma serotonin and urinary excretion of 5 hydroxy indole acetic acid were raised. The child is asymptomatic four years after diagnosis.

The past decade has seen major developments in the understanding of endocrine tumours of gut (apudomas) and the related clinical syndromes resulting from excessive secretion of gut hormones.' Apudomas may be classified ${ }^{1}$ into those containing enterochromaffin cells (secreting serotonin, substance $\mathbf{P}$, histamine, kallikrein, motilin, protaglandin, and kinin) and non-enterochromaffin cells (secreting gastrin, glucagon, insulin, somatostatin, and vasoactive intestinal polypeptide). Resultant hypersecretory syndromes including gastrinoma, vipoma, glucagonoma, somatostatinoma, and carcinoid syndrome are rare and still scarcely considered in the differential diagnosis of childhood diarrhoea.

\section{Case report}

A 9 year old girl, the second child of healthy unrelated Scottish parents, presented with intermittent watery diarrhoea which she had suffered since the age of 5 months. A physical examination at 11 months had shown no abnormality. Passage of loose watery stools continued at three to four weekly intervals, but the patient thrived.

Despite three admissions to hospital for investigation of paroxysmal abdominal pain and diarrhoea at 6,8 , and 9 years, no abnormality could be found; nor did she suffer diarrhoea during several weeks in hospital. Abrupt mood changes were noted but although a psychiatric opinion was sought no abnormality was found.

Six months later the patient was again admitted to hospital with attacks of colicky abdominal pain, six to eight times daily, relieved by passing a brown watery stool. There was frequent vomiting and weight loss, her height and weight were on the third centile, and there was generalised wasting. The abdomen was distended and hyperresonant, the liver was $2 \mathrm{~cm}$ below the costal margin, and a $4 \times 4$ $\mathrm{cm}$ epigastric mass was palpable. 


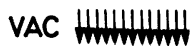

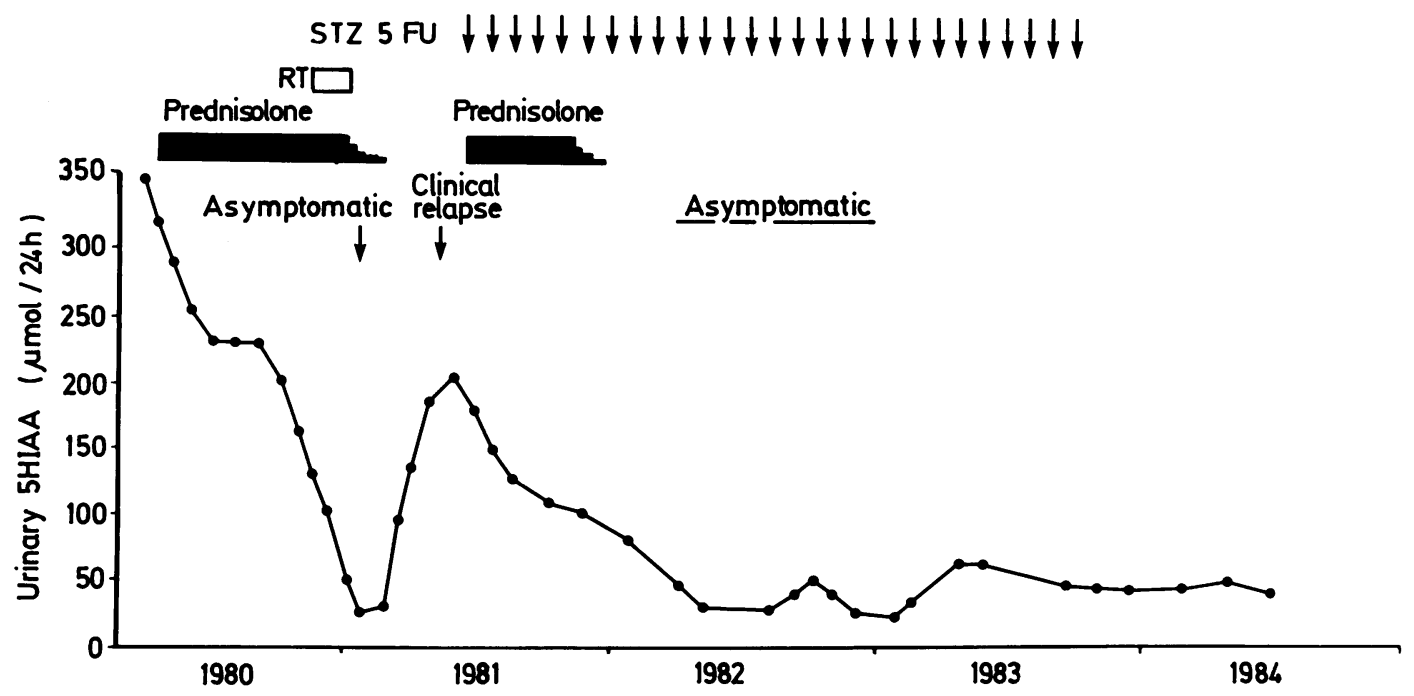

Figure Effect of treatment on urinary 5-hydroxy indole acetic acid (5 HIAA) excretion and symptoms.

$\mathrm{V}=$ vincristine: $\mathrm{A}=$ adriomycin; $\mathrm{C}=$ cyclophosphamide; $\mathrm{STZ}=$ streptozotocin; $5 \mathrm{FU}=5$ fluorouracil; $\mathrm{RT}=$ radiotherapy.

Routine investigations were repeatedly normal on each admission to hospital. In addition, complete gastrointestinal barium studies; jejunal histology; brush border enzymes; plasma vasoactive intestinal polypeptide, glucagon, gastrin, and somatostatin; and urinary homovanillic acid and 4 hydroxy-3methoxy-mandelic acid values were normal.

At laparotomy, enlarged lymph glands were found at the head of pancreas anterior to the aorta and inferior vena cava. Multiple umbilicated nodules were seen throughout both lobes of liver. Histological examination of biopsy material showed the features of a malignant carcinoid tumour with well defined clusters of uniform small to medium sized cells involving liver and largely replacing the paraaortic lymph nodes. Acinar differentiation was evident and ultrastructural studies showed tumour cells containing numerous darkly staining, membrane bound, secretory granules 120 to $140 \mathrm{~nm}$ in diameter.

After the operation facial flushing was seen and the patient suffered episodes of abdominal pain. Twenty four hour urinary excretion of 5 hydroxy indole acetic acid was $314 \mu \mathrm{mol}$ (normal less than 50 $\mu \mathrm{mol})$ and the blood serotonin concentration was $727 \mu \mathrm{g} / \mathrm{l}$ (normal 100 to $250 \mu \mathrm{g} / \mathrm{l}$ ). Screening for multiple endocrine neoplasia was negative. Isotope liver scan showed two filling defects in the inferior surface of the left lobe. Computed tomographic abdominal scan confirmed a $3 \times 4 \mathrm{~cm}$ mass at the head of pancreas.

Treatment is shown in the Figure. Daily prednisolone $(5 \mathrm{mg}$ ) and fortnightly streptozotocin $10 \mathrm{mg} / \mathrm{kg}$, with 5-fluorouracil, $15 \mathrm{mg} / \mathrm{kg}$, resulted in the resolution of symptoms and biochemical remission after six months. Prednisolone was withdrawn after a further nine months: streptozotocin and 5-fluorouracil were continued for two years without adverse effect and were stopped when a potentially nephrotoxic dose level was reached.

At 14 years of age the patient is asymptomatic and thriving and has had no treatment for one year. There is no hepatomegaly and the epigastric mass is impalpable, having slightly diminished in size on computed tomogram to $2.4 \times 4 \mathrm{~cm}$.

\section{Discussion}

Carcinoid tumours are unusual in children, occurring mostly in the appendix and usually discovered at routine appendicectomy. ${ }^{2}$ Carcinoid syndrome is excessively rare-only four cases have been reported. . $^{-5}$

The clinical features of the syndrome (paroxysms of flushing, abdominal pain, diarrhoea, borborgymi, weight loss, skin rashes, bronchospasm, valvular heart disease, and psychiatric symptoms) result from the release of pharmacologically active mediators 
(serotonin, histamine, kinins) from tumour tissue. ${ }^{1}$ Symptoms arise when massive liver replacement by tumour results in impaired metabolism of amines and peptides (as in the case of intestinal carcinoid) or less commonly when a primary tumour (stomach, pancreas, bronchus, ovary, testis) secretes its products directly into the circulation. Urinary 5hydroxy indole acetic acid excretion is usually raised and may be a useful marker for the effectiveness of treatment. ${ }^{6}$ Where the diagnosis is made pre-operatively, removal of the primary tumour or resection of hepatic matastases, or both, or hepatic artery ligation ${ }^{6}$ should be considered.

Response to chemotherapy is variable: 5fluorouracil and streptozotocin, singly or in combination, are most commonly used. ${ }^{6}$ Dramatic improvement of symptoms during steroid treatment and after radiotherapy ${ }^{3}$ has been reported, and symptomatic relief may be achieved with drugs which block the synthesis, release, or pharmacological action of serotonin or kinins. ${ }^{6}$

Our patient may have had carcinoid syndrome since infancy: before diagnosis at 9 years, the paroxysmal symptoms and negative investigations were misinterpreted as implying a functional disorder. Despite surgical failure to remove the carcinoid tumour it is striking that the patient is asymptomatic three years after chemotherapy. The prognosis for this child remains uncertain. In the event of relapse, the question of whether further chemotherapy would be valuable arises since recurrence may proceed slowly as in adults.

Carcinoid syndrome should be considered in obscure cases of childhood diarrhoea, even if, as in this case, other classic features are lacking.

We thank Dr M L N Willoughby, Dr J R M McKenzic, and the Department of Medical Illustration, Yorkhill Hospital.

\section{References}

${ }^{1}$ Grahame-Smith DG. The carcinoid syndrome. In: Truelove SC, Lee E, eds. Topics in gastroenterology Vol 5. Oxford: Blackwe!l Scientific Publications, 1977:285-312.

2 Anderson A, Bergdahl L. Carcinoid tumour of the appendix in children. Acta Chir Scand 1977;143:173-5.

${ }^{3}$ Chow CW, Sane S, Campbell PE, Carter RF. Malignant carcinoid tumours in children. Cancer 1982;49:802-11.

${ }^{4}$ Field JL, Adamson LF, Stoeckle HE. Review of carcinoids in children. Functioning carcinoid in a 15 year old male. Pediatrics 1962;29:953-60.

5 Biorck G, Axen D, Thomson A. Unusual cyanosis in a boy with congenital pulmonary stenosis and tricuspid insufficiency: fatal outcome after angiocardiography. Am Heart J 1952;44:143-8.

${ }^{6}$ Moertel CG. Treatment of the carcinoid tumour and malignant carcinoid syndrome. J Clin Oncol 1983;1:727-40.

Correspondence to Dr M D King, Department of Child Health, Royal Hospital for Sick Children, Glasgow G3 8SJ.

Received 9 November 1984 